\title{
The Correlations Between Epidemiological and Clinical Characteristics, laboratory tests and CT Scan reports in the diagnosis of cases 2019 novel coronavirus pneumonia. A Diagnostic Accuracy Study
}

\author{
Bobby Branson ${ }^{1}$, Ramin Tavakoli², Mansoor Khaledi ${ }^{3}$, Seyed Mohammad Shafiee ${ }^{4}$, Hamed \\ Afkham $^{3}$, and Shahdokht Rastegar ${ }^{2}$ \\ ${ }^{1}$ Comenius University in Bratislava Jessenius Faculty of Medicine in Martin \\ ${ }^{2}$ Ahvaz Jondishapour University of Medical Sciences \\ ${ }^{3}$ Shahed University \\ ${ }^{4}$ Shiraz University of Medical Sciences
}

January 17, 2021

\begin{abstract}
Background: The role of laboratory parameters and the relationship of them with radiology reports, CT scan and clinical outcomes in screening of COVID-19 patients not been definitely established, but this disease presented a major challenge in the field of clinical tests, radiology reports, clinical outcomes that help to monitoring and treatment COVID-19 disease. Methods: This study was performed on 340 suspected COVID-19 patients, who presented to Chamran Hospital, Shiraz University of Medical Sciences Shiraz, Iran from 20 February to 31 August, 2020. Information each patient's will be completed using a data collection forms based on records. The evaluation of lungs involvement in CT scan and their relationship with laboratory indicator including biochemical and hematological factors, is the best scale for the severity and prognosis of Covid 19 patients. Results and Conclusion: The findings of this study indicated ALT, AST, CRP, NEU, LDH, and Urea have very good accuracy in predicting cases with positive RT-PCR for COVID-19, respectively. In this study we shown the correlation of clinical and laboratory findings with CT-based quantitative score of pulmonary involvement in COVID-19 pneumonia and attempted that our findings could be usable to development future clinical research associated with COVID-19 infection and show the relationship of reports CT scan and clinical outcomes in the diagnosis and severity of patients with COVID-19.
\end{abstract}

The Correlations Between Epidemiological and Clinical Characteristics, laboratory tests and CT Scan reports in the diagnosis of cases 2019 novel coronavirus pneumonia. A Diagnostic Accuracy Study

Running Title: The correlations between Clinical Findings, laboratory tests and CT reports in the diagnosis of with COVID-19 patients.

Bobby Branson ${ }^{1}$, Ramin Tavakoli ${ }^{2}$, Mansoor Khaledi ${ }^{3}$, Seyed Mohammad Shafiee ${ }^{2}$, Hamed Afkham ${ }^{3}$, Shahdokht Rastegar ${ }^{4 *}$

1. Biomedical Centre Martin, Department of surgery, Jessenius Faculty of Medicine in Martin, Comenius University in Bratislava, Martin, Slovakia.

2. Department of Biochemistry, Faculty of Medicine, Student Research Committee, Shiraz University of Medical Sciences, Shiraz, Iran. 
3. Department of Microbiology, Faculty of Medicine, Shahed University, Tehran, Iran

4. Toxicology Research Center, Department of Biochemistry, School of Medicine, Student Research Committee, Ahvaz Jundishapur University of Medical Sciences, Ahvaz, Iran.

Corresponding author: Shahdokht Rastegar*. Department of Biochemistry, Toxicology Research Center, School of Medicine, Student Research Committee, Ahvaz Jundishapur University of Medical Sciences, Ahvaz, Iran. Tel: +989389237286, E-mails: h.a.university.ac@gmail.com, Shahdokht63rastegar@gmail.com

Address: Department of Biochemistry, Toxicology Research Center, School of Medicine, Student Research Committee, Ahvaz Jundishapur University of Medical Sciences, Ahvaz, Iran.

\begin{abstract}
Background: The role of laboratory parameters and the relationship of them with radiology reports, CT scan and clinical outcomes in screening of COVID-19 patients not been definitely established, but this disease presented a major challenge in the field of clinical tests, radiology reports, clinical outcomes that help to monitoring and treatment COVID-19 disease.
\end{abstract}

Methods: This study was performed on 340 suspected COVID-19 patients, who presented to Chamran Hospital, Shiraz University of Medical Sciences Shiraz, Iran from 20 February to 31 August, 2020. Information each patient's will be completed using a data collection forms based on records. The evaluation of lungs involvement in CT scan and their relationship with laboratory indicator including biochemical and hematological factors, is the best scale for the severity and prognosis of Covid 19 patients.

Results and Conclusion: The findings of this study indicated ALT, AST, CRP, NEU, LDH, and Urea have very good accuracy in predicting cases with positive RT-PCR for COVID-19, respectively. In this study we shown the correlation of clinical and laboratory findings with CT-based quantitative score of pulmonary involvement in COVID-19 pneumonia and attempted that our findings could be usable to development future clinical research associated with COVID-19 infection and show the relationship of reports CT scan and clinical outcomes in the diagnosis and severity of patients with COVID-19.

Keywords: Clinical Outcomes, laboratory findings, coronaviruses, COVID- 19 infection, CT scan reports.

\title{
What is already known about this topic?
}

1- The investigation of laboratory parameters and CT scan reports have an important role and very good accuracy in predicting cases with positive RT-PCR for COVID-19 clinical outcomes and screening of COVID19 patients.

\section{What does this article add?}

1- In this study we investigated the correlation of clinical and laboratory findings with CT-based quantitative score of pulmonary involvement in COVID-19 pneumonia, that we realized radiology reports and CT scan findings may be predictive of patient's outcome and had a correct correlate with laboratory findings and disease severity, the use of laboratory findings in besides of CT scan reports as a first line test can be beneficial to speed up diagnostic and therapeutic workflow.

\section{INTRODUCTION}

The new covid-19 coronavirus, a severe infectious disease with rapid transmission to humans, was discovered in December 2019, and its outbreak was declared by the World Health Organization as a public health emergency and has now claimed the lives of millions worldwide ${ }^{1}$. Diagnosis of Covid - 19 disease is performed using polymerase chain reaction tests, chest CT scans, and ELISA test kits ${ }^{2,3}$. Research published since 2019 claims that chest CT scans, along with polymerase chain reaction tests and other laboratory parameters, have the highest efficiency in detecting Covid $19^{4,5}$. Therefore, chest CT scan and radiological reports have been proposed as an adjunct to polymerase chain reaction tests as a complementary method ${ }^{6}$. No findings can completely confirmed or rule out the possibility of COVID-19 ${ }^{7}$. Some observational studies have been 
partially characterized Clinical, laboratory, and imaging features of Covid-19, but until now few systematic study on relate these features to each other have been published to date ${ }^{8}$. Laboratory tests scales in the most patients with COVID-19 showed a significant changes in serum pro-inflammatory cytokine levels and blood biochemical markers such as interleukins, TNF-a, LDH, AST, ALT BUN, Creatinine, D-dimers, platelet count, cardiac troponin, renal markers WBC, HB and Lymphocytes (according to figure. 1) ${ }^{9,10}$. Evaluation of clinical features and radiological reports in severe cases of COVID-19 will help in the initial prognosis, accurate diagnosis and treatment of patients with Covid-19 ${ }^{11-13}$. It was detecting that there are differences in the abundance of metabolic markers and biochemistry indicators in COVID-19 patients than healthy, but there is a question about the subject whether change in blood biochemistry markers are associated with CT-scan findings and the severity of COVID-19 disease? Most of the articles published in the field have reported the epidemiological and clinical characteristics of patients with COVID-19 disease, but data regarding the relationship of laboratory characteristics with CT-scan findings of infected individuals are limited ${ }^{14-16}$. However, the evaluation of lungs involvement in CT scan and their relationship with laboratory indicator including biochemical and hematological factors, is the best scale for the severity and prognosis of Covid 19 patients ${ }^{17}$. Various studies described the epidemiological and clinical characteristics of hospitalized patients ${ }^{10,18-20}$. However, there is a limited number of detailed analysis on blood chemicals in discharged patients. In this study, we attempted that our findings could be usable to development future clinical research associated with COVID-19 infection. In concluded, this study aimed to evaluate laboratory parameters and the relationship them with $\mathrm{CT}$ scan reports and clinical outcomes in the diagnosis of patients with COVID-19.

\section{METHODS}

This study is a cross sectional study conducted of collected data on patients of all age groups, this study was performed on 340 suspected COVID-19 patients, who presented to Chamran Hospital, Shiraz University of Medical Sciences Shiraz, Iran from 20 February to 31 August 2020 and in this study after obtaining permission from the ethics committee and approving the research plan, the clinical and laboratory findings of covid-19 patients were extracted from the electronic medical records of the patients. Information each patient including the evaluation of lungs and kidney involvement in CT scan and sonography and their relationship with laboratory indicator including biochemical and hematological factors for the severity and prognosis of Covid 19 disease was investigated by a trained team of physicians. The data about clinical symptoms, medicine treatment and laboratory parameters were collected by patient record, and based on the doctor's diagnosis and CT scan reports and PCR test of throat Secretions. Nasal and pharyngeal swab samples and sputum were obtained from patients on admission. We retrospectively analyzed and evaluated the epidemiological history, comorbidity, vital signs, and symptoms obtained from electronic medical records. The data collection forms were reviewed independently by two experienced physicians, and the severity of the disease is determined by the need for ICU, sepsis, involvement of the lungs and other organs, intubation, ventilation, death. The information of each case was completed using a data collection form based on the records recorded in the patient file and the examination of radiological photographs, and a sample of the data collection form was attached. Other variables studied include the need for ICU hospitalization and the time of hospitalization from the onset of symptoms to hospitalization and the need for intubation and ventilation. Problems include ARDS, Renal Injury, Septic Shock and Ventilator Associated Injury. In this study, CT scan reports are examined and the consistency of patients' deterioration based on these reports with the patient's fate in the case is examined. Inclusion criteria included, all positive coronavirus patients referred to chamran Hospital, who need to be hospitalized. Exclusion criteria included, deaths before admission to the hospital and positive coronavirus patients or those who for some reason refused to visit the hospital. This project has been approved by the research ethics committee with the code of IR.SUMS.REC. 1399.S605 and project number of 99-10-24-13415 by Shiraz University of Medical Sciences in Shiraz, Iran.

\subsection{Statistical analyses}

The collected data was entered and analyzed through the data collection form in SPSS software version 20. For descriptive analysis, mean and standard deviation will be used for quantitative variables and frequency 
and frequency percentage will be used for qualitative variables. To evaluate the effect of each variable as a univariate test, independent two-sample t-test, one-way ANOVA and chi-square will be performed for quantitative and qualitative factors, respectively. Also, the study of the effect of variables is multivariate modeled using logistic regression model and significance is considered at the level of 0.05. The MannWhitney U test was utilized to compare significant differences among continuous data. All statistical tests were two tailed, and the $\mathrm{p}$ value less than 0.05 was considered statistically significant.

\section{RESULTS}

Demographic characteristics of all patients was listed in Table 1. The mean age of patients was 41.7, the median age 63 years (range 32-69) for critical patients which $65 \%$ were male and $34 \%$ were female (one-way ANOVA, p < 0.0001). Almost 198 patients (58\%) were aged [?] 38 years. Generally, 183 (53\%) patients had one or two types of chronic diseases, such as cancer, immune system defects, coronary heart disease (CHD), hypertension (HBP) and diabetes (DM). Hypertension 154 (45.29 \%), Cardiovascular Disease 90 (26.47\%) and Diabetes 178 (52.35\%) were the most common Coexisting Conditions. According to the results of this study, 28 patients $(9 \%)$ of patients had familial infection or familial clusters. There was significant difference among patients by their epidemiology history. The patients indeed presented significantly different on the most common symptoms like Fever, Dyspnea, Muscular pain, Fatigue, Shortness of Breath, Chill, Dry Cough and Diarrhea (Table 1) (one-way ANOVA, $\mathrm{p}=0.001$ ). Furthermore, the mean length of hospital stay in these patients were 14.12 days that in the critically ill patients took average 2.08 days longer than total average levels. From the 340 cases with the suspicion of COVID-19 infection, 230 patients (164 males, 76 females with a positive RT-PCR test for covid-19). The most common clinical manifestations were fever, coughing, Muscular pain and dyspnea. In this study change CRP levels were found in patients and increased D-dimer levels were found in patients and decreased lymphocyte count was observed in 98 patients according to Table 2 Summary of Changes in Biomarkers Seen in Severe COVID-19 Infection. Change clinical in biochemistry parameters patients are summarized in Table 2. The results of this study show that the white blood cell (WBC) and neutrophil cell numbers were significantly higher and platelets and HB severely lower and a quite distinguishable difference on the blood biochemical test at different patients. Among patients in people with underlying diseases and high-risk groups showed significantly higher levels of serum ALT, AST, LDH, than other healthy. According to Table 2 ALT, CRP, LDH, and Urea had very good precision in predicting cases with positive RT-PCR for COVID19. The result of this study RT-PCR for COVID-19 patients was positive in $252(74 \%)$ cases and negative in $88(25 \%)$. Based on the CDC clinical scoring for covid-19 infection (10), $150(44.11 \%)$ were classified as mild, $118(34.70 \%)$ as severe, and 72 patients (21.17\%) as critical.

The results of this study ( Table 3) in CT features, scoring, screening patients show the most common patterns of disease included GGO, observed in 152 patients (44.7\%), followed by followed by crazy-paving pattern $68(20 \%)$ and parenchymal consolidations in $78(22.9 \%)$ (Fig. 2). Features and characteristics related to CT were found as follows: fibrosis $(\mathrm{n}=57 ; 16.76 \%)$, sub pleural lines $(\mathrm{n}=43 ; 12.46 \%)$, pleural effusion $(\mathrm{n}=28 ; 8.23 \%)$, precordial effusion $(\mathrm{n}=8 ; 2.35 \%)$, and mediastinal lymphadenopathy $(\mathrm{n}=27$; $7.94 \%)$, COPD thinking of ILD, ILS $(\mathrm{n}=30 ; 8.82 \%)$. Findings of this study also shown lobar involvement, lesion distribution, and localization in pulmonary parenchyma. Pathological involvement in the left lower lobe (LLL) in 157 patients (46.17\%) was most common and right lower lobe (RLL) in 143 patients (42.05 \%). The mean of involvement lung lobes and CT scores were show in (Fig. 3) (Table.4).

According to the average global CT score was $12.3 \pm 11.1$. All patient did show parenchymal involvement at $\mathrm{CT}$ reports and there are not any patient therefor scored as 0 . In comparisons between lung lobes, the mean CT score was significantly higher in RLL than in ML and RUL $(\mathrm{p}<0.0001)$ and the mean CT score was significantly higher in LLL than in LUL $(\mathrm{p}<0.0001)$ (Fig. 3), also the distribution of parenchymal abnormalities in pathological findings were posterior in 118 patients $(34.70 \%)$ and anterior in 68 patients $(20$ $\%)$. In the 50 patients (16.8\%), there was involvement of both anterior and posterior areas. Regarding the results of this study and investigation of CT features, demonstrated the GGO pattern was most prevalent in early-phase disease and late-phase disease, while crazy-paving and consolidation patterns were most common in late-phase, also fibrosis were significantly common in late-phase. The pleural effusion and lymphadenopa- 
thy in patients were rarely observed in late-phase. CT score in late-phase was significantly higher than in early-phase patients $(\mathrm{p}<0.0001)$. CT score between age range groups statistically significant difference was found $(\mathrm{p}=0.0018)$, in this way CT score was significantly higher in age range $>75$ than in other age groups $(\mathrm{p}=0.001)$.

Furthermore, the results of this study indicated statistically significant correlations between CT score with SAA $(\mathrm{p}<0.0001, \mathrm{r}=0.4314)$, LDH $(\mathrm{p}<0.0001, \mathrm{r}=0.3214)$, Cardiac troponin $(\mathrm{p}<0.0001, \mathrm{r}=0.6714)$, Renal biomarkers Urea \& creatinine $(\mathrm{p}<0.0001, \mathrm{r}=0.3314)$, CRP $(\mathrm{p}<0.0001, \mathrm{r}=0.6314)$, D-dimer $(\mathrm{p}<0.0001$, $\mathrm{r}=0.6427)$, lymphocyte count $(\mathrm{p}=0.0001, \mathrm{r}=0.1630)$ levels. Univariate and multivariate analyses of 340 patients in this study show that 48 patients $(11.17 \%$ ) died during a mean follow-up of $14.1 \pm 4.8$ days (range 1-26 days), all of which indicated at least one or more of the previously mentioned underlying diseases. The mortality rate was significantly higher in patients [?]75 years old $(\mathrm{n}=39 ; 11.47 \%)$ and among critical patients $(12 / 12 ; 100 \%)$. The univariate analysis CT score in this patients indicated a higher risk of death in patients with a CT score [?]18 (HR, 8.23; 95\% CI, 2.17-25.63; $<<0.0001)$, and significantly correlated with increase of age (HR,1.02;95\% CI,1.01-1.21; $\mathrm{p}=0.001)$, HDL (HR, 1.01; 95\% CI, 1.03-1.07; $\mathrm{p}<0.001)$, Cardiac troponin (HR, 1.003; 95\% CI, 1.10-1.00; p<0.001), Urea (HR, 1.06; 95\% CI, 1.03-1.07; p <0.001), creatinine (HR, 1.01; 95\% CI, $1.00-1.01 ; \mathrm{p}<0.001)$ CRP (HR, 1.06; 95\% CI, 1.03-1.07; $\mathrm{p}<0.001)$ and D-dimer levels (HR,1.011; 95\% CI,1-1.08.001; $\mathrm{p}=0.0001)$. Table 5 show the correlations between Clinical Findings, laboratory tests and CT reports.

In this study we investigated the correlation of clinical and laboratory findings with CT-based quantitative score of pulmonary involvement in COVID-19 pneumonia, that we realized CT scan findings may be predictive of patients 'outcome and had a correct correlate with laboratory findings and disease severity.

Table 1 Demographic and clinical characteristics.

Demographic characteristics

Sex: (n \%) Male Female

Age: (n \%) 0-25 26-50 51-75 >75

Symptoms: Fever Coughing Dyspnea Diarrhea Headache Muscular pain

Underlying disease Hypertension Cardiovascular Disease Obesity or Hyperlipidemia Diabetes Chronic obstr

Table 2 Comparing the laboratory findings of COVID-19 patients between Positive and Negative cases.

\begin{tabular}{lllll}
\hline Parameters & Total $(\mathrm{n}=340)$ & RT-PCRforCOVID-19 & RT-PCRforCOVID-19 & P- value \\
\hline & & Positive $(\mathrm{n}=252)$ & Negative $(\mathrm{n}=88($ & \\
WBC & $6962.8 \pm 2127$ & $4343 \pm 1002$ & $6994 \pm 1982$ & $<0.0001$ \\
NEU & 48.1 & 62.2 & 45.7 & $<0.0001$ \\
LYM & 45.8 & 39.1 & 52.1 & $<0.0001$ \\
POSITIVE CRP a & 39 & 57 & 29 & $<0.0001$ \\
ESR & $27.2 \pm 8.6$ & 32 & 20 & 0.486 \\
AST & $\mathbf{2 8 . 6} \pm 8.6$ & $32.1 \pm 8.01$ & $21.24 \pm 8.1$ & 0.533 \\
ALT & $32 \pm 7.1$ & $38.8 \pm 2.1$ & $25.2 \pm 5.9$ & 0.926 \\
LDH & $385.6 \pm 125$ & $471.2 \pm 110.2$ & $361.6 \pm 94.1$ & 0.0001 \\
SAA & $28.2 \pm 8.01$ & $36.1 \pm 8.5$ & $24.8 \pm 4.9$ & 0.0001 \\
Urea & $43.12 \pm 32.41$ & $66.81 \pm 53.83$ & $42.78 \pm 33.42$ & 0.001 \\
Creatinine & $1.39 \pm 0.95$ & $1.69 \pm 0.95$ & $1.97 \pm 9.32$ & 0.877 \\
CK-MB & $899.35 \pm 255.21$ & $1033.45 \pm 1754.19$ & $216.38 \pm 474.51$ & 0.0001 \\
D-dimer & $0.4 \pm 1.5$ & $0.5 \pm 2.9$ & $0.3 \pm 0.7$ & 0.0001 \\
Blood urea Nitrogen & 35 & $58-70$ & $8-24$ & 0.001 \\
\hline
\end{tabular}


WBC: white blood cell; NEU: Neutrophil; LYM Lymphocyte AST: aspartate aminotransferase; ALT: alanine aminotransferase; LDH lactate dehydrogenase; ESR: erythrocyte sedimentation rate; CRP: $\mathrm{C}$ reactive protein; CK-MB, creatine kinase-muscle and brain type; SAA; serum amyloid $\mathrm{A}$

Table 3 Frequency of main patterns and features in covid-19 patients.

CT features in COVID -19 patients

Main CT pattern Ground glass opacity Crazy paving Consolidation Mixed GGO and consolidation

Related features Fibrosis Sub pleural lines Pleural effusion precordial effusion mediastinal Lymphadenopath

Table 4 Frequency of involvement of each lobe with related CT score.

Categories

Lung Lobe Right upper lobe (RUL) Middle lobe (ML) Right lower lobe (RLL) Left upper lobe (LUL) Left

Table 5 The correlations between Clinical Findings, laboratory tests and CT reports.

laboratory tests

CT SCORE

WBC NEU LYM

POSITIVE CRP a ESR

AST ALT LDH SAA Urea \& Creatinine TROPONIN CK-MB D-dimer Blood urea Nitrogen

r P- value

$\mathrm{r}=0.1520 \mathrm{r}$

$\mathrm{r}=0.6314 \mathrm{r}=$

$\mathrm{r}=0.3517 \mathrm{r}=$

\section{DISCUSSION}

According the findings of the present study, it seems that there is a significant relationship between chest CT scan characteristics and change clinical parameters with outcomes of COVID-19 cases. Based on this results ground Glass Opacity (GGO) and consolidation were the most common chest CT scan findings, which was match to other researches ${ }^{21,22}$. The most common location of abnormalities was lower zone involvement that observed more than upper zone, which was consistent with findings of other studies ${ }^{19,23}$. An investigation carried out on other patients show that CT reports will be more obvious, when revealed longer the onset of symptoms ${ }^{24}$. In this study radiological patterns have correlation with the stages of the development disease, which was in match to the studies ${ }^{24,25}$. The findings of this study indicated ALT, AST, CRP, NEU, LDH, and Urea have very good accuracy in predicting cases with positive RT-PC R for COVID-19, respectively. Also, liver injury is more prevalent in severe cases compared to mild cases of COVID-19. This study was similar to study Chen et al. Another study indicated that 2-11\%of patients with COVID-19 had ALT and AST levels during progression of COVID-19 disease ${ }^{19}$. In comparison to the normal range, in patients with positive RT-PCR COVID19, the rate of WBC and LYM counts was decreased and NEU counts increase, which is in line with another study ${ }^{26}$. The COVID -19 Virus extensive through the respiratory system and infected immune cells and causing changes in number of peripheral white blood cells such as lymphocytes ${ }^{27}$. Various studies proposed the substantial decrease in the number of lymphocytes following infection with the coronavirus that influence immune cells and prevent immune action ${ }^{28}$. Also, others report show that high neutrophil count and LDH level in COVID-19 patients were autonomous predictors of an adverse clinical outcome ${ }^{29}$. The results of laboratory parameters in this study, such as ALT, CRP, AST, LDH, and NEU indicated that there could be used to foretell the presence of COVID-19 disease, while WBC were poor predicted of the disease. This information is match to findings reported by Wang et al. ${ }^{30}$ and Gao et al. ${ }^{31}$. Thus, some laboratory parameters can use to screening cases with positive RT-PCR 
forCOVID-19. Overall, Clinical course of the COVID-19 disease is unforeseeable, due to the heterogeneity of its manifestations and multi organ failure. At present, there are no prognostic biological markers to identify Covid 19 patients and estimate their associated mortality. It seems that predicting CT scan reports of disease progression and its relationship with laboratory-clinical findings may be useful in patient triage and symptomatic treatment of patients ${ }^{32}$. In this study we used a previously CT score for confirming this presumption, that it was based on the lobar Involvement expanse of as reported by Pan et al ${ }^{33}$. The results of the study shown correlation CT scoring with laboratory parameters, age, dyspnea at admission and the presence of pre-existing comorbidities like coronary arteries and diabetes. Information of mortality rate in this study have approved the outstanding importance of age and its rate in patients older than 75 years. our finding in the study substantially assessment CT parenchymal reports and may reflect short and high-term outcome through direct visualization of anatomic damages than with non-specific laboratory parameters and inflammatory biomarkers. Based on clinical criteria provided by the WHO ${ }^{34}$ and as expected, finding of this study indicated the correlation of disease severity with CT reports and laboratory parameters, so CT scores were significantly confirmed high correlation between imaging findings and clinical stages, however, the diagnostic role of CT scan and its correlation to laboratory parameters is controversial and debated. Although, the use of CT is as a first line test, but highly sensitive imaging method like CT associated with specific clinical parameters, might be beneficial to speed up diagnostic and therapeutic workflow.

\section{Conclusion}

Considering the significant difference in CT scoring and laboratory parameters, can hope to model or predict the results of coronavirus testing based on routine laboratory tests. In conclusion, finding of this study shown the correlation of clinical and laboratory findings with CT-based quantitative score of pulmonary involvement in COVID-19 pneumonia and our findings could be usable to development future clinical research associated with COVID-19 infection and show relationship of CT scan reports and clinical outcomes in the diagnosis and severity of patients with COVID-19. Thus, expected future studies can indicated better clarify impact on clinical decision-making and larger clinical trials.

\section{Limitation}

Differences between Iran's National Guideline for COVID-19 and guidelines of other countries, in addition to usual limitations of cross sectional studies, were among the most important limitations of this study. We performed a retrospective analysis evaluation in a relatively limited cohort of patients, but the severity of the current health care emergency implies that a prospective evaluation would have been extremely complex and longer to complete.

\section{ACKNOWLEDGEMENTS}

The authors would like to thank Shiraz University of Medical Sciences, Shiraz, Iran, Clinical Biochemistry Research Center of Shiraz University of Medical Sciences, Shiraz, Iran, Department of Laboratory Sciences, School of Paramedical Sciences, Ahvaz Jundishapur University of Medical Sciences, Ahvaz, Iran for the Specialized Commissioning in this paper.

\section{DISCLOSURE}

The authors declare that they have no known competing financial interests or personal relationships that could have appeared to influence the work reported in this paper.

\section{AUTHORCONTRIBUTIONS}

BB, RT, MK, SR: design of study. BB, MK, HA, SMS: acquisition of data. BB, SR, RT, MK, : evaluation of data, preparation of the manuscript. BB, RT, MK, SR : assessment of data. All authors read and approved the final manuscript.

\section{DATA AVAILABILITY STATEMENT}


The data that supports the findings of this study are available in the article Further enquiries can be directed to the corresponding authors.

\section{ORCID}

Bobby Branson https://orcid.org/0000-0002-0844-156X

Ramin Tavakoli https://orcid.org/0000-0002-6030-2217

Mansoor Khaledi https://orcid.org/0000-0002-1576-5968

Hamed Afkham https://orcid.org/0000-0002-1110-6447

Shahdokht Rastegar https://orcid.org/0000-0001-9947-5900

\section{Funding}

This research did not receive any specific grant from funding agencies in the public, commercial, or not-forprofit sectors.

\section{Abbreviations}

ARDS Acute respiratory distress syndrome CDC Center of Disease Control COVID-19 Coronavirus disease 2019

CRP C-reactive protein GGO Ground glass opacityLLL Left lower lobe LUL Left upper lobe MLMiddle lobe

RLL Right lower lobe RT-PCR Real-time polymerase chain reaction RUL Right upper lobe

TAT Turnaround time WHO World Health Organization

\section{REFERENCES}

1 Manuel, B. et al. 2019-Novel Coronavirus (2019-NCOV): Estimating the case fatality rate-A word of caution. Swiss Med Wkly. 1502020.

2 Sohrabi, C. \& Alsafi, Z. O” Neill. N., Khan, M., Kerwan, A., Al-Jabir, A., et al, 71-76 2020.

3 Saberiyan, M. et al. An Overview on the Common Laboratory Parameter Alterations and their Related Molecular Pathways in Screening for COVID-19 Patients. Clin Lab . 662020.

$4 \mathrm{Ai}$, T. et al. Correlation of chest CT and RT-PCR testing in coronavirus disease 2019 (COVID-19) in China: a report of 1014 cases.Radiology . 2006422020.

5 Lupia, T. et al. 2019-novel coronavirus outbreak: A new challenge.J Glob Antimicrob Resist . 2020.

6 https://acaai.org/news/important-covid-19-information-those-asthma-andor-allergies

7 Ebisawa, M. et al. PHYSICIAN BRIEF.

8 Bohn, M. K. et al. Molecular, serological, and biochemical diagnosis and monitoring of COVID-19: IFCC taskforce evaluation of the latest evidence. Clin. Chem. Lab. Med (CCLM). 12020.

9 Russell, C. D., Millar, J. E. \& Baillie, J. K. Clinical evidence does not support corticosteroid treatment for 2019-nCoV lung injury. The Lancet. 395, 473-475 2020.

10 Huang, C. et al. Clinical features of patients infected with 2019 novel coronavirus in Wuhan, China. The lancet . 395, 497-506 2020.

$11 \mathrm{Xu}, \mathrm{Z}$. et al. Pathological findings of COVID-19 associated with acute respiratory distress syndrome. Lancet Respir Med. 8, 420-422 2020.

12 Bai, Y. et al. Presumed asymptomatic carrier transmission of COVID-19. Jama . 323, 1406-1407 2020. 
13 D'Amico, F., Baumgart, D. C., Danese, S. \& Peyrin-Biroulet, L. Diarrhea during COVID-19 infection: pathogenesis, epidemiology, prevention and management. Clin Gastroenterol Hepatol . 2020.

14 Yuan, J. et al. The correlation between viral clearance and biochemical outcomes of 94 COVID-19 infected discharged patients. J. Inflamm. Res . 1-8 2020.

15 Deng, X. et al. Blood biochemical characteristics of patients with coronavirus disease 2019 (COVID-19): a systemic review and meta-analysis. Clin. Chem. Lab. Med (CCLM) 12020.

$16 \mathrm{Hu}, \mathrm{Q}$. et al. Association between severity of COVID-19 and clinical and biochemical characteristics: a cross-sectional study. 2020.

17 Kermali, M., Khalsa, R. K., Pillai, K., Ismail, Z. \& Harky, A. The role of biomarkers in diagnosis of COVID-19-A systematic review.Life Sci . 1177882020.

18 Wang, D. et al. Clinical characteristics of 138 hospitalized patients with 2019 novel coronavirus-infected pneumonia in Wuhan, China.Jama. 323, 1061-1069 2020.

19 Chen, N. et al. Epidemiological and clinical characteristics of 99 cases of 2019 novel coronavirus pneumonia in Wuhan, China: a descriptive study. The Lancet . 395, 507-513 2020.

$20 \mathrm{Xu}, \mathrm{X} .-\mathrm{W}$. et al. Clinical findings in a group of patients infected with the 2019 novel coronavirus (SARSCov-2) outside of Wuhan, China: retrospective case series. bmj . 3682020.

21 Ye, Z., Zhang, Y., Wang, Y., Huang, Z. \& Song, B. Chest CT manifestations of new coronavirus disease 2019 (COVID-19): a pictorial review. Eur Radiol . 1-9 2020.

22 Yuan, M., Yin, W., Tao, Z., Tan, W. \& Hu, Y. Association of radiologic findings with mortality of patients infected with 2019 novel coronavirus in Wuhan, China. PloS one. 15, e0230548 2020.

23 Wong, H. Y. F. et al. Frequency and distribution of chest radiographic findings in COVID-19 positive patients. Radiology . 2011602020.

24 Bernheim, A. et al. Chest CT findings in coronavirus disease-19 (COVID-19): relationship to duration of infection. Radiology . 2004632020.

25 Pan, F. et al. Time course of lung changes on chest CT during recovery from 2019 novel coronavirus (COVID-19) pneumonia.Radiology . 2020.

26 Zhang, C., Shi, L. \& Wang, F.-S. Liver injury in COVID-19: management and challenges. Lancet Gastroenterol Hepatol. 5, 428-430 2020.

27 Shi, H. et al. Radiological findings from 81 patients with COVID-19 pneumonia in Wuhan, China: a descriptive study. Lancet Infect Dis . 2020.

$28 \mathrm{Qu}$, R. et al. Platelet-to-lymphocyte ratio is associated with prognosis in patients with coronavirus disease-19. J Med Virol . 2020.

29 Lei, J., Li, J., Li, X. \& Qi, X. CT imaging of the 2019 novel coronavirus (2019-nCoV) pneumonia. Radiology . 295, 18-18 2020.

30 Tsui, P. T., Kwok, M. L., Yuen, H. \& Lai, S. T. Severe acute respiratory syndrome: clinical outcome and prognostic correlates.Emerg. Infect. Dis . 9, 10642003.

31 Gao, Y. et al. Diagnostic utility of clinical laboratory data determinations for patients with the severe COVID-19. J Med Virol . 2020.

32 Rubin, E. J. et al. Harrington DP, Hogan JW, Gatsonis C, Baden LR, Hamel MB: The urgency of care during the Covid-19 pandemic - learning as we go. In.: (Mass Medical Soc, 2020).

33 Littrup, P. J. et al. Cryotherapy for breast fibroadenomas. Radiology 234, 63-72 2005. 
34 Simpson, S. et al. Radiological Society of North America Expert Consensus Statement on Reporting Chest CT Findings Related to COVID-19. Endorsed by the Society of Thoracic Radiology, JACR , and RSNA. Radiology: Cardiothoracic Imaging 2, e200152 2020.

35 Francone, M. et al. Chest CT score in COVID-19 patients: correlation with disease severity and shortterm prognosis. Eur Radiol. 30, 6808-6817 (2020).

\section{Figure legends}

Fig. 1 Different CT score of RLL involvement in COVID-19 pneumonia on axial, sagittal, and coronal images. $0 \%$ of RLL lobe involvement (a); $<5 \%$ of RLL involvement (b); $20 \%$ of RLL involvement (c); $40 \%$ of RLL lobe involvement (d); $70 \%$ of RLL involvement (e); >75\% of RLL involvement (f) ${ }^{35}$.

Fig. 2 Chest CT findings of COVID-19 pneumonia on axial images. GGO (A); crazy-paving pattern (GGO with superimposed inter- and interlobular septal thickening) (B); consolidation (C); GGO, crazy-paving pattern and consolidation (D).

Fig. 3 Lobar CT scores of patients Covid-19, data are expressed as mean value \pm SD (\% of occurrences of involvement for each lobe) $(* * * * \mathrm{p}<0.0001)$. RUL, right upper lobe; ML, middle lobe; RLL, right lower lobe; LUL, left upper lobe; LLL, left lower lobe.

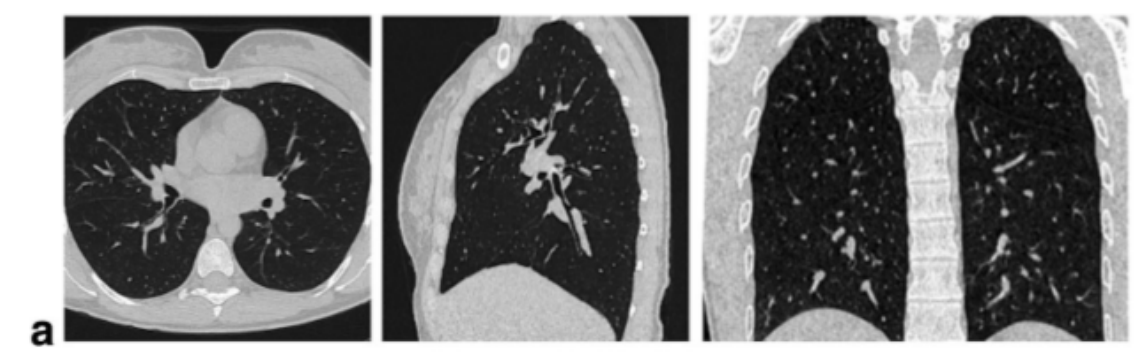

CT Score $0.0 \%$ of RLL

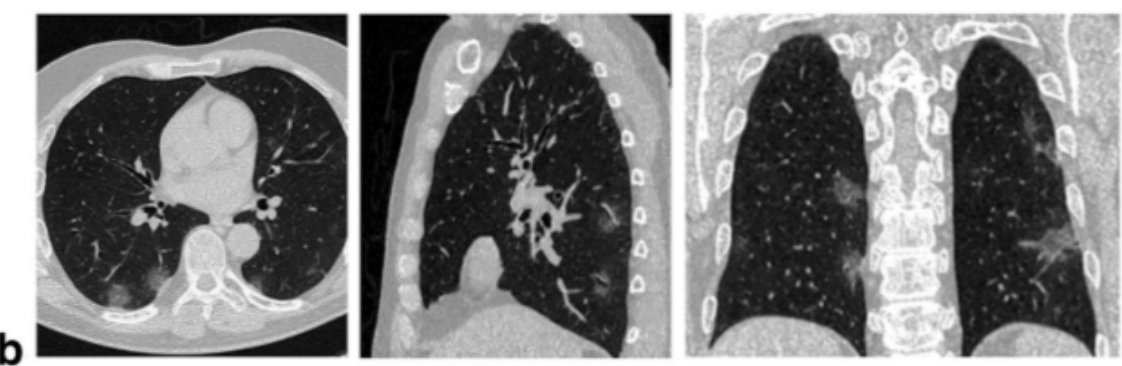

CT Score 1. $<5 \%$ of RLL

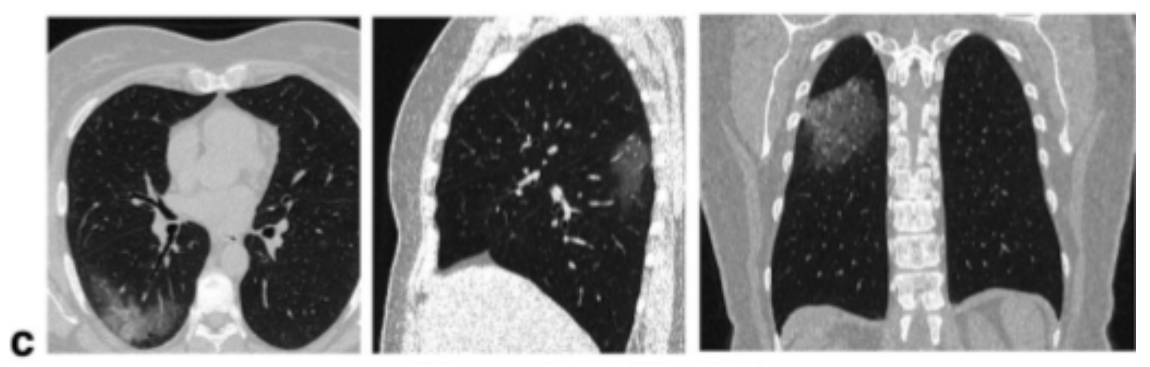


CT Score 2. 5-25\% of RLL

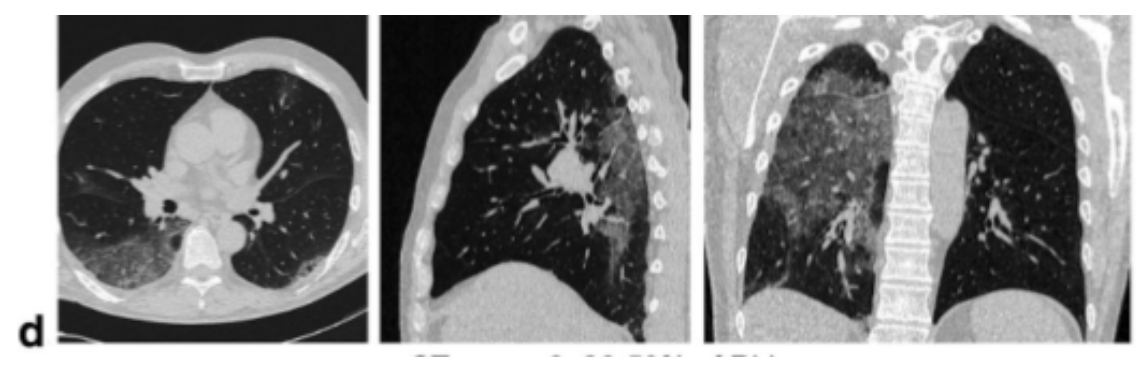

CT Score 3. 26-50 \% of RLL

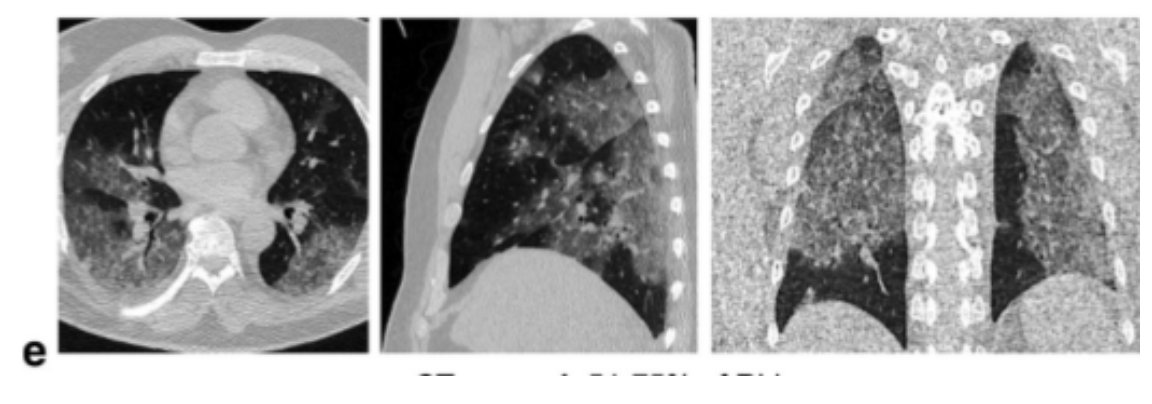

CT Score 4. 51-75\% of RLL
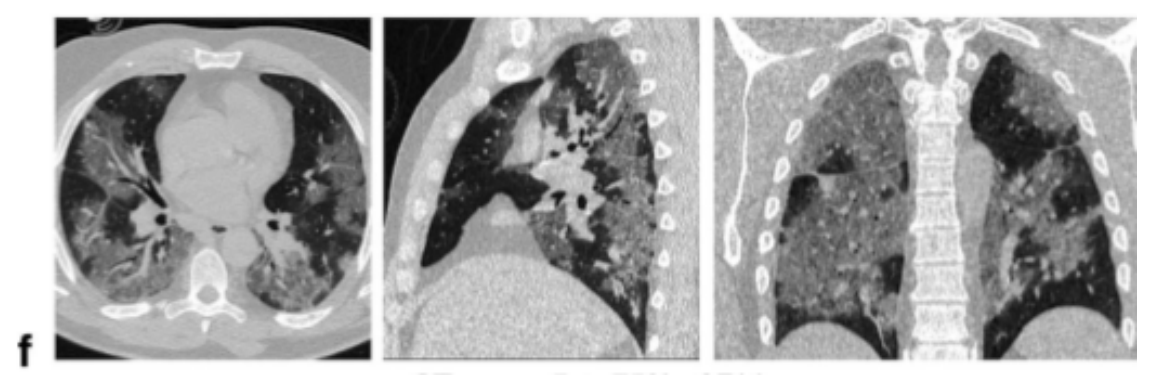

CT Score 5. 76-100 \% of RLL

Fig. 1 Different CT score of RLL involvement in COVID-19 pneumonia on axial, sagittal, and coronal images. $0 \%$ of RLL lobe involvement (a); <5\% of RLL involvement (b); $20 \%$ of RLL involvement (c); $40 \%$ of RLL lobe involvement (d); $70 \%$ of RLL involvement (e); $>75 \%$ of RLL involvement (f) [21]. 

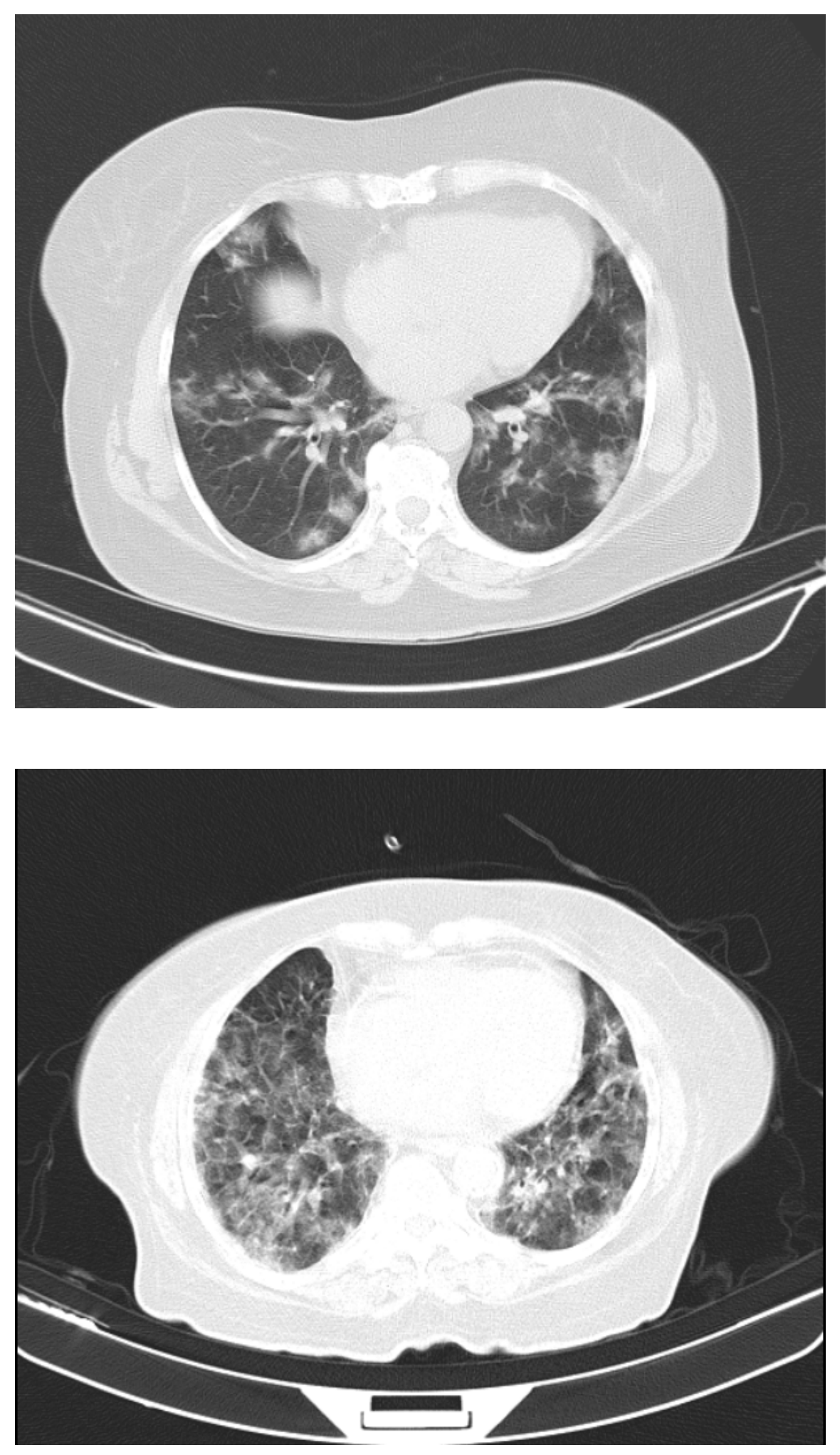

A 
B
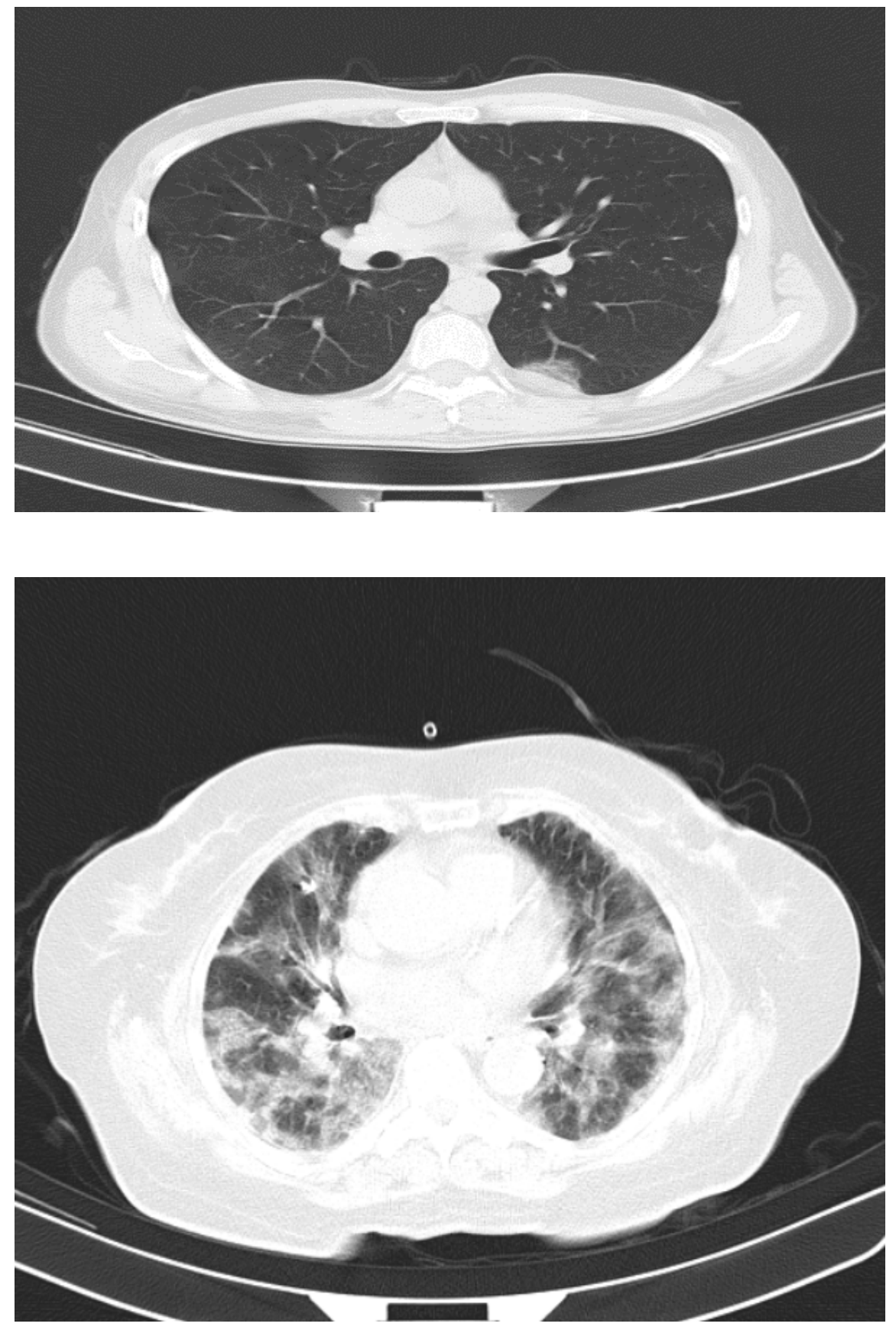

C

D

Fig. 2 Chest CT findings of COVID-19 pneumonia on axial images. GGO (A); crazy-paving pattern (GGO with superimposed inter- and interlobular septal thickening) (B); consolidation (C); GGO, crazy-paving pattern and consolidation (D). 


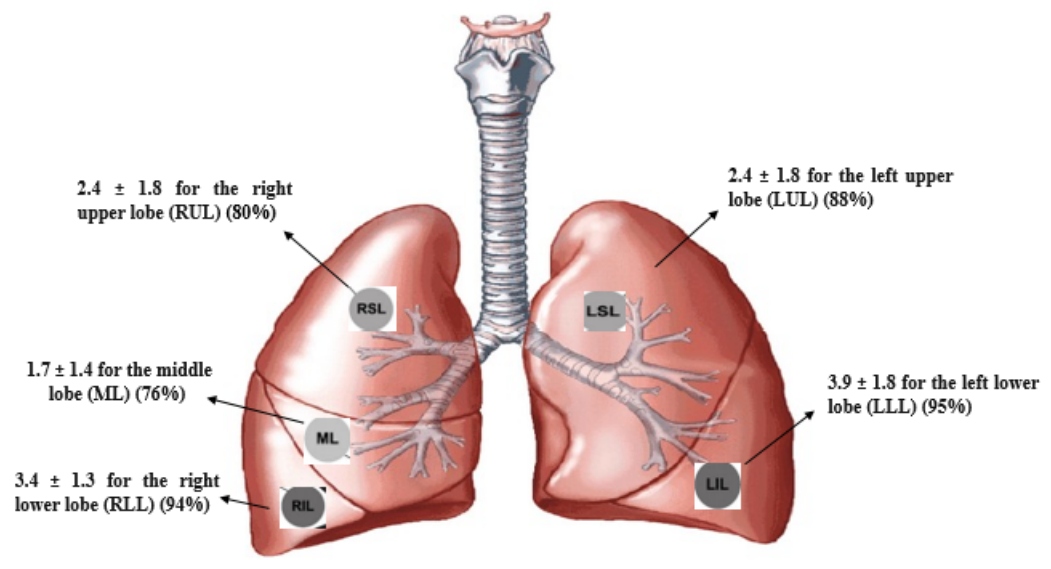

Fig. 3 Lobar CT scores of patients Covid-19, data are expressed as mean value \pm SD (\% of occurrences of involvement for each lobe) $(* * * * p<0.0001)$. RUL, right upper lobe; ML, middle lobe; RLL, right lower lobe; LUL, left upper lobe; LLL, left lower lobe.

\section{Hosted file}

Figures.pdf available at https://authorea.com/users/390012/articles/504469-the-correlationsbetween-epidemiological-and-clinical-characteristics-laboratory-tests-and-ct-scanreports-in-the-diagnosis-of-cases-2019-novel-coronavirus-pneumonia-a-diagnosticaccuracy-study 